\title{
Las TICs y el desempeño docente en el proceso de E-A en la facultad de Ciencias- Unasam-Huaraz, 2014
}

\section{ICTs and teaching performance in the E-A process at the faculty of science-Unasam- Huaraz, 2014}

Jonhson Valderrama $\mathrm{A}^{1}$ y Marcela Saldaña M. ${ }^{2}$

\begin{abstract}
RESUMEN
El objetivo de la investigación, determinar la relación entre las tecnologías de la información y la comunicación (TICs) y el desempeño docente en el proceso de enseñanza aprendizaje de la facultad de ciencias - UNASAM - Huaraz, 2 014. La investigación de tipo descriptivo, método cuantitativo y diseño correlacional; se aplicaron dos instrumentos: encuesta sobre las TICs y otra sobre desempeño docente en el proceso de enseñanza-aprendizaje validados por dos expertos, aplicados a una muestra poblacional de 108 docentes de la facultad de ciencias. Después de analizar los resultados, se afirma que existe relación directamente y altamente significativa $(\mathrm{p}=0,0000<0,05)$ entre las tecnologías de la información y la comunicación (TICs) y el desempeño docente en el proceso de enseñanza- aprendizaje; quedando corroborado la hipótesis afirmativa.
\end{abstract}

Palabras clave: TICs, desempeño docente, proceso de E-A, redes.

\begin{abstract}
The objective of the research is to determine the relationship between information and communication technologies (ICTs) and teaching performance in the teaching-learning process of the faculty of science - UNASAM - Huaraz, 014. Descriptive type research, quantitative method and correlational design; Two instruments were applied: survey on ICTs and another on teacher performance in the teaching-learning process validated by two experts, applied to a population sample of 108 teachers of the faculty of science. After analyzing the results, it is affirmed that there is a direct and highly significant relationship $(\mathrm{p}=0.0000<0.05)$ between information and communication technologies (ICTs) and teaching performance in the teaching-learning process; The affirmative hypothesis is corroborated.
\end{abstract}

Keywords: ICTs, teaching performance, E-A process, networks.

\footnotetext{
${ }^{1}$ Universidad Nacional Santiago Antúnez de Mayolo, Huaraz, Perú. Email: marcelasaldanamiranda@hotmail.com
}

${ }^{2}$ Universidad Nacional Santiago Antúnez de Mayolo, Huaraz, Perú. Email: jonhson_val@ hotmail.com 


\section{INTRODUCCIÓN}

Hoy por hoy, los estudiantes que asisten a las universidades tienen un apoyo para su proceso de E-A, que son las TICs, mediante las redes sociales, como el Facebook (2004), WhatsApp (2009), Twitter (2006), según (J. y. Gómez 2003), que les permiten entrar al mundo de la búsqueda de información, investigación, que les facilita sus aprendizajes; las redes sociales están revolucionando la manera de comunicarse, entretenerse, de estudiar e incluso de informarse; por lo cual deben los docentes deben hacer uso de las TICs en su desempeño docente en el proceso de enseñanza aprendizaje.

Las TICs favorece el desempeño docente en el proceso de E-A, motivo por el cual los docentes deben incorporar en su quehacer cotidiano en el aula, las TICs se están convirtiendo poco a poco en un instrumento indispensable en las Universidades, este recurso permite nuevas posibilidades para la docencia universitaria abriendo canales de comunicación logrando así, intercambiar ideas, al razonamiento del porqué de lo que se dijo entre los integrantes de un grupo, favoreciendo el trabajo en equipo; y la toma de decisiones.

La sociedad actual llamada, Sociedad de la información, demanda cambios en los sistemas educativos de forma que éstos se tornen más flexibles y accesibles, menos costosos y a los que han de poderse incorporar los ciudadanos en cualquier momento de su vida. La Universidad, para responder a estos desafíos, debe revisar sus referentes actuales y promover experiencias innovadoras en los procesos de EA, apoyados en las TICs. El énfasis debe hacerse en la docencia, en los cambios de estrategias didácticas de los docentes universitarios, en los sistemas de comunicación y distribución de los materiales de aprendizaje, en lugar de enfatizar la disponibilidad y las potencialidades de las tecnologías.

En este marco, el país desde el año 2002 se inició el proceso de integración de las TIC en el sistema educativo público estatal a través del Proyecto Huascarán y desde el año 2007 a través de la Dirección General de Tecnologías Educativas del Ministerio de Educación, con la finalidad que las TIC “mejoren la calidad de la educación secundaria para que los estudiantes alcancen una formación integral que comprenda la consecución de logros de aprendizaje y una sólida formación en valores".

Debido a estos avances de la inserción de las TICs en la educación, consideramos los trabajos de investigación, como es el caso de, (García 2011). Influencia de las TIC en el Aprendizaje Significativo. España. Tesis doctoral. El autor llega a la conclusión de que las TIC influyen adecuadamente y presentan beneficios en los aprendizajes significativos. Lógicamente las TICs favorecen el desempeño docente en el proceso de enseñanza - aprendizaje, como lo garantiza el trabajo de investigación de (Castañeda 2011) . Tecnologías digitales y el proceso de enseñanza- aprendizaje en la educación secundaria. Madrid, tesis doctoral. 
Las tecnologías de información y comunicación presentan muchas ventajas como recurso útil en el desempeño docente dentro de los procesos de enseñanza y aprendizaje, cuyas dimensiones son las redes, los terminales y los servicios.

Para, (Chiavento 2007); define el desempeño laboral como toda acción realizada o ejecutada por un individuo, en respuesta, de todo aquello de lo que es responsable y que será medio en base a su ejecución, claro está que el proceso enseñanza-aprendizaje como un sistema de comunicación intencional que se produce en un marco institucional y en el que se generan estrategias encaminadas a provocar el aprendizaje.

Las TICs, hoy en día son recursos didácticos valiosos y ya forman parte de la dinámica educativa, por lo que los docentes tienen el reto de conocerlos e incorporarlos a su desempeño docente en el proceso de enseñanza - aprendizaje. El acceso a recursos TICs programas y materiales en el aula puede ofrecer un entorno mucho más rico para el aprendizaje y una experiencia docente más dinámica. La utilización de contenidos digitales de buena calidad enriquece el aprendizaje y puede, a través de simulaciones y animaciones, ilustrar conceptos y principios que de otro modo serían muy difíciles de comprender para los estudiantes. Hay algunas evidencias de aprendizajes enriquecidos y profundizados por el uso de TICs. Las TICs son fuertemente motivadoras para los estudiantes y brindan encuentros de aprendizaje más activos; en el aprendizaje basado en proyectos y en trabajos grupales, permite el acceso a recursos y a expertos que llevan a un encuentro de aprendizaje más activo y creativo tanto para los estudiantes como para los docentes. La evaluación del aprendizaje es una dimensión clave para cualquier dominio de aprendizaje personalizado. (Morrissey 2003).

En la universidad actual nos enfrentamos a estudiantes digitales que demandan un nuevo tipo de enseñanza. Los universitarios han crecido bajo la influencia del audiovisual y de la red. Las nuevas herramientas tecnológicas (redes sociales, blogs, plataformas de vídeo, etcétera) les han dado el poder de compartir, crear, informar y comunicarse, convirtiéndose en un elemento esencial en sus vidas, (M. Gómez 2012).

Es pertinente destacar que las características de las tecnologías de información y comunicación presentan muchas ventajas como recurso útil en el desempeño docente dentro de los procesos de enseñanza y aprendizaje entre los que se puede destacar la información, acceso a gran cantidad de información sobre diferentes ámbitos; flexibilidad instruccional, ritmo de aprendizaje adecuándose a las necesidades diversas que requiere el alumno y exige el aula; complementariedad de códigos de comunicación, aplicaciones multimedia empleando diversos códigos de comunicación; aumento de la motivación y de actividades colaborativas y cooperativas entre los intervinientes alumnos o instituciones por medio de la red; pseudo información y sobrecarga de información; obtención de mucha información en poco tiempo, 
ocasionando el efecto de saturación cognitiva; dependencia tecnológica, el uso adecuado de los medios tecnológicos y la construcción de conocimientos evitará la dependencia tecnológica en la construcción de significados; del aprendizaje autónomo, de la dotación de sentido, la comprensión y el aprender a aprender con un mayor valor, al "saber cómo" sobre el "saber qué o sobre qué". Los medios y recursos a ser utilizados deben estar subordinados al proceso educativo, y no el proceso educativo subordinado a los medios y recursos a ser utilizados. (Sancho 1995).

Asimismo, dentro de las aulas universitarias, las TICs, juegan un papel importante. Según (Salinas 2004), “...En nuestras universidades podemos encontrar multitud de experiencias de «enseñanza virtual», «aulas virtuales», etc., incluidos proyectos institucionales aislados de la dinámica general de la propia entidad que, aunque loables, responden a iniciativas particulares y, en muchos casos, pueden ser una dificultad para su generalización, al no ser asumidas por la organización como proyecto global. Así, este tipo de iniciativas particulares no hace sino poner de manifiesto la rigidez de las estructuras universitarias para integrar en su funcionamiento cotidiano la utilización de las TIC en los procesos de enseñanza-aprendizaje. Pero se requiere la participación activa y motivación del profesorado, pero se necesita, un fuerte compromiso institucional. La cultura universitaria promueve la producción, la investigación, en detrimento muchas veces de la docencia y de los procesos de innovación en este ámbito. Y, sin embargo, procesos de este tipo parecen ser los que oxigenarán de alguna forma a las universidades...”.

La educación está frente a un nuevo paradigma, (Bralavsky 1999); señala que no sólo se remite a lo científico y tecnológico -como un aspecto del ámbito sociocultural y político- sino que también, se fundamenta en las bases epistemológicas que lo sustentan. Estos cambios impactan tanto en el plano institucional y cultural como en el plano didáctico y metodológico. “...Las nuevas generaciones se ven inmersas en un contexto mediático y tecnológico más competitivo que demanda de forma inmediata nuevas habilidades y conocimientos del uso de TIC..." (Meza 2002) La realidad educativa actual concibe al constructivismo como una propuesta epistemológica que surge en oposición al positivismo del conductismo y el procesamiento de la información; además, se basa en la concepción que la realidad es una construcción interna, propia del individuo. Dicha forma de ver el constructivismo, indica (Sánchez 2007), está justificada desde la perspectiva del uso de las tecnologías de información y comunicación para la construcción del conocimiento.

El constructivismo al igual que otras teorías, proporciona principios sobre el aprendizaje que tienen importantes aplicaciones para la construcción de entornos tecnológicos de enseñanza. Una de estas implicaciones es la necesidad de introducir el aprendizaje dentro de contextos reales y significativos. Las 
TICs pueden ser muy útiles en la obtención de información, planteamiento de interrogantes y en el aprendizaje colaborativo. El uso de programas de simulación y estrategia, los multimedia e hipermedia, así como las redes cibernéticas, son herramientas básicas en entornos de aprendizaje bajo principios constructivistas. La Informática debe ayudar a crear situaciones y ofrecer instrumentos que permitan a los alumnos sacar el máximo partido de sus capacidades cognitivas. La telemática permite la aplicación de un modelo de enseñanza constructivista, ya que proporcionan a los alumnos oportunidades para comunicarse, investigar, debatir asuntos y trabajar colaborativamente. Las TICs por sí solas no permiten desarrollar un entorno constructivista de aprendizaje, pero proporcionan un medio en el que se incrementa la posibilidad de poner en práctica este modelo de enseñanza. Así permiten a los alumnos comunicarse, investigar, debatir puntos de vista y trabajar colaborativamente.

La revolución de los medios ha supuesto una transformación social semejante a lo que en su día produjeran la escritura o la imprenta, ya que incide directamente sobre el conocimiento humano. Ahora se refleja dos expectativas educativas distintas: por un lado, tenemos a los informáticos, interesados en aprender informática, y, en el otro, a los docentes, interesados en el uso de la informática para la educación. Durante todo el desarrollo de este trabajo estaré abordando los temas que, a nuestro juicio, son de gran importancia para conocer las tecnologías de la información y la comunicación y su uso como una herramienta para fortalecer el desempeño docente.

Este informe concibe al constructivismo como una propuesta epistemológica que surge en oposición al positivismo del conductismo y el procesamiento de la información; además, que se basa en la concepción que la realidad es una construcción interna, propia del individuo. Dicha forma de ver el constructivismo, indica (Sánchez 2007), está justificada desde la perspectiva del uso de las tecnologías de información y comunicación para la construcción del conocimiento.

Esta investigación se justifica, porque permite que nuestros docentes de la Facultad de Ciencias de la UNASAM incorporen las TICs en el desempeño diario del proceso de enseñanza aprendizaje, como un recurso didáctico que les va a permitir obtener aprendizajes significativos, ya que en los cursos de ciencias básicas existe considerable número de desaprobados, que posiblemente uno de los factores sea la formación de los docentes o no hacen usos de las bondades que tienen las TICs. El uso de las TICs, le permitirá establecer procedimientos para la mejora educativa de la labor docente, reduciendo el fracaso, la deserción y permitiendo acabar con los métodos didácticos que exigen esfuerzos inútiles tanto a estudiantes como a profesores y por último elevar los niveles de excelencia académica.

En la Facultad de Ciencias de la Universidad Nacional "Santiago Antúnez de Mayolo" (UNASAM), cuenta con 108 docentes; los cuales no tienen formación pedagógica, por lo que la mayoría el desempeño docente en el proceso de enseñanza aprendizaje lo desarrollan sus clases siguiendo la secuencia de textos 
generalmente. Por estas razones creemos que es justificable considerar dicho trabajo de investigación como una propuesta las Tecnologías de la Información y la Comunicación y el desempeño docente en el proceso de enseñanza aprendizaje de la Facultad de Ciencias - UNASAM-Huaraz, 2 014, con la única finalidad de mejorar el desempeño docente en el proceso de enseñanza-aprendizaje, cuyo objetivo consiste en determinar la relación que existe entre las Tecnologías de la Información y la Comunicación (TICs) y el desempeño docente en el proceso de E - A de la Facultad de Ciencias - UNASAM-Huaraz, 2014.

\section{MATERIALES Y MÉTODOS}

La investigación de tipo descriptivo, método cuantitativo y diseño correlacional; se aplicó dos instrumentos, el primero sobre TICs, que constaron de 15 preguntas; y el segundo instrumento sobre desempeño docente con 30 preguntas validados por dos expertos, aplicados a una muestra poblacional de 108 docentes de la Facultad de Ciencias.

\section{Población y muestra}

Tabla 1. Población Muestral

\begin{tabular}{cccc}
\hline Facultad de Ciencias & Población & Muestra \\
\hline- & Directivos & 06 & \\
& Docentes & 102 & 108 \\
& & 108 & 108 \\
\hline
\end{tabular}

Fuente: Oficina de personal de la UNASAM 10/07/2014

La población que se tendrá en cuenta en el presente estudio son los docentes de la Facultad de Ciencias - UNASAM-Huaraz 2014, de 108 docentes.

\section{Técnicas e instrumentos de recolección de datos}

Las técnicas, que se usa para recolectar la información, es una encuesta, cuyos instrumentos fue mediante un cuestionario el cual estará conformado por un conjunto de preguntas cuyo origen serán los indicadores que medirán las dimensiones de cada variable.

Para obtener el nivel de fiabilidad fueron tomados en cuenta 30 preguntas la primera sección del cuestionario abarca el área que corresponde a Desempeño docente en el Proceso de enseñanzaaprendizaje, y por último la segunda sección con 15 preguntas abarca el área de Tecnologías de la información y la comunicación (TICs). Teniendo con una confiabilidad del instrumento se da gracias al 
coeficiente de alfa de cronbach se obtuvo un coeficiente de 0,827(en conjunto) lo que indica que el instrumento tiene un alto nivel de confiablidad.

Para realizar el análisis de datos utilizamos la estadística descriptiva y para la contrastación de hipótesis mediante la prueba de Ji- cuadrado de Pearson

\section{RESULTADOS}

En concordancia con los objetivos planteados en esta investigación, se muestra los resultados a través de tablas y gráficos, que son el reflejo de la opinión de los docentes de la Facultad de Ciencias - UNASAM sobre "Tecnologías de la información y la comunicación y el desempeño docente en el proceso de enseñanza -aprendizaje de la Facultad de Ciencias - UNASAM - Huaraz, 2 014”. Estos resultados se presentan en la forma siguiente: Validación de la hipótesis, que viene hacer el cruce de las Tecnologías de la información y de la comunicación y el Desempeño Docente en el proceso de Enseñanza Aprendizaje.

Tabla 2. TICs y el desempeño docente en el proceso de E-A

\begin{tabular}{llcccccc}
\hline & & \multicolumn{3}{c}{ Deficiente } & \multicolumn{2}{c}{ Regular } & \multicolumn{2}{c}{ Excelente } \\
\cline { 3 - 8 } & & $\mathrm{n}$ & $\%$ & $\mathrm{n}$ & $\%$ & $\mathrm{~N}$ & $\%$ \\
\multirow{3}{*}{ TICs } & 8 & $7,4 \%$ & 11 & $10,2 \%$ & 0 & $0,0 \%$ \\
& Deficiente & 13 & $12,0 \%$ & 65 & $60,2 \%$ & 1 & $0,9 \%$ \\
& Regular & 13 & $0,0 \%$ & 7 & $6,5 \%$ & 3 & $2,8 \%$ \\
\hline
\end{tabular}

Fuente: Base de datos de resumen

Descripción: En la Tabla 2, podemos apreciar que el 60,2\% de los docentes es regular en el manejo de las TICs y en su desempeño docente en el proceso de enseñanza-aprendizaje; el $12 \%$ utilizan en forma regular pero a su vez tienen deficiencia en el desempeño docente en el proceso de enseñanza-aprendizaje; el 10,2\% muestran deficiencia en uso de TICs pero su desempeño docente en el proceso de enseñanzaaprendizaje, es regular; el 7,4\% tienen deficiencia en el manejo de TICs y en el desempeño docente en el proceso de enseñanza-aprendizaje; así mismo el 6,5\% manejan excelentemente las TICs y a su vez su desempeño docente en el proceso de enseñanza-aprendizaje es regular; el 2,8\% son excelentes en el manejo de las TICs y en su desempeño docente en el proceso de enseñanza-aprendizaje; y el 0,9\% utilizan las TICs regularmente y a su vez el desempeño docente en el proceso de enseñanza-aprendizaje es excelente.

Tabla 3. Grado de significancia

Chi - cuadrada $\quad X_{c}^{2}=29,291 \quad$ gl. $=4 \quad \mathbf{P}=0,000^{*, \mathrm{~b}, \mathrm{c}<0.05 \text { Significativo }}$


Así mismo, en la Tabla 3, se observa que el valor de la Chi-Cuadrado es $X_{c}^{2}=20,398$ con nivel de significancia de $\mathrm{p}=0,0001$ siendo menor al 5\% siendo altamente significativo, la cual quiere decir que existe relación directa y significativa entre las Tecnologías de la Información y la Comunicación (TICs) y el desempeño docente en el proceso de enseñanza - aprendizaje de la facultad de ciencias - UNASAMHuaraz, 2014.

Se afirma entonces que existe relación directa y altamente significativa $(\mathrm{P}<0,05)$ entre las Tecnologías de la Información y la Comunicación (TICs) y el desempeño docente en el proceso de enseñanzaaprendizaje de la facultad de ciencias - UNASAM-Huaraz, 2014.

\section{DISCUSIÓN}

En lo referente al objetivo general "Determinar la relación que existe entre las Tecnologías de la Información y la Comunicación (TICs) y el Desempeño Docente en el proceso de E - A de la Facultad de Ciencias - UNASAM-Huaraz, 2014”; en la tabla 2, podemos apreciar que el 60,2\% de los docentes es regular en el manejo de las TICs y en su desempeño docente en el proceso de enseñanza-aprendizaje, mientras que el $12 \%$ utilizan en forma regular pero a su vez tienen deficiencia en el desempeño docente en el proceso de enseñanza-aprendizaje, el 10,2\% muestran deficiencia en uso de TICs pero su desempeño docente en el proceso de enseñanza-aprendizaje, es regular; mientras que 7,4\% tienen deficiencia en el manejo de TICs y en el desempeño docente en el proceso de enseñanza-aprendizaje; así mismo el 6,5\% manejan excelentemente las TICs y a su vez su desempeño docente en el proceso de enseñanza-aprendizaje, es regular; mientras que el 2,8\% son excelentes en el manejo de las TICs y en su desempeño docente en el proceso de enseñanza-aprendizaje; y con un menor porcentaje $0,9 \%$ utilizan las TICs regularmente y a su vez el desempeño docente en el proceso de enseñanza-aprendizaje, es excelente.

Dichos resultados guardan relación con los que sostiene (Sancho 1995), al destacar que las características de las tecnologías de información y comunicación presentan muchas ventajas como recurso útil en el desempeño docente dentro de los procesos de enseñanza y aprendizaje entre los que se puede destacar la información variada, acceso a gran cantidad de información sobre diferentes ámbitos; flexibilidad instruccional ritmo de aprendizaje adecuándose a las necesidades diversas que requiere el alumno y exige el aula; concordando con el estudio de (Castañeda 2011). Donde indica que la incorporación de las TICs en el Proceso de enseñanza- aprendizaje en la educación secundaria es necesario como un recurso didáctico generando una metodología creativa- colaborativa. Se concluye que 
por experiencia como docente universitario podemos decir que existe relación entre las Tecnologías de la Información y la Comunicación (TICs) y el Desempeño Docente en el proceso de enseñanza aprendizaje de la facultad de ciencias, tal como se puede apreciar en los resultados obtenidos según la encuesta realizada a los docentes.

\section{CONCLUSIONES}

La investigación nos ha permitido determinar la relación entre las tecnologías de la Información y la comunicación y el desempeño docente en el proceso de E-A de la Facultad de Ciencias de la UNASAM2014, el cual resultó en una relación muy significativa, ya que guarda relación directa las tecnologías de la Información y la comunicación y el desempeño docente en el proceso de E-A de la Facultad de Ciencias.

De acuerdo a la hipótesis planteada resulto cierta, ya que el trabajo de investigación muestra una existencia de evidencia empírica que el desempeño docente en el proceso de E-A está relacionada con las Tecnologías de la Información y la comunicación en la Facultad de Ciencias de la UNASAM-Huaraz 2014.

\section{REFERENCIAS BIBLIOGRÁFICAS}

Balarín, Luisa. 2012. Las políticas TIC en los sistemas educativos de América Latina: caso Perú. Argentina: UNICEF.

Bralavsky, Cecilia. 1999.Rehaciendo escuelas hacia un nuevo paradigma en la educación latinoamericana. Buanos Aires, Santillana.

Castañeda, María. 2011.Tecnologías digitales y el proceso de enseñanza - aprendizaje en la educación secundaria. Tesis Doctoral, Madrid: Fundación santillana.

Gómez , Marisol. 2012. El uso académico de las redes sociales en universitarios. http://www.revistacomunicar.com/pdf/preprint/38/14-PRE-13426.pdf. consultado el 28-012015.

Meza, Perez y De la Barreda, Berenice. 2002. Comunidades virtuales de aprendizaje como herramienta para el apoyo de la labor docente. Madrid: Tecnoscoipo.

Chiavento, Idalberto. 2007. Introducción a la Teoría general de la Administración. Mc Graw Hill.

García , Felix. 2011.«Influencia de las TIC en el Aprendizaje Significativo.» Tesis doctoral, Madrid.

Gómez, Juan y Redondo, Cristina. 2003. «Las redes sociales como fuente de conocimiento en la enseñanza Primaria.» Universidad de Barcelona,: 1-10. 
Morrissey, Jerome.2003. «El uso de TIC en la enseñanza y el aprendizaje.» Cuestiones y Desafios,: 110.

Salinas, Jesús. 2004. «Innovación docente y uso de las TIC en la enseñanza universitaria.» Revista Universitaria y Sociedad de Conocimiento,: 1-16.

Sánchez, María. 2007. «Elearning e innovación educativa basada en TICs y web social. Recuperado el 06 del 2015.»: http://creatic.innova.unia.es/redes-sociales.

Sancho, Juana. 1995. «¿El medio es el mensaje o el mensaje es el medio?»Revista electrónica Píxelbit,: 3-4. 